\title{
Protocole de prise en charge des troubles de l'hémostase chez les patients positifs pour le VHC et le VIH, faut-il les transfuser avant une intervention chirurgicale ?
}

\author{
Vidaud C, Radoi L, Krouma A, Nguyen T, Poidatz E \\ Service d'Odontologie, Hôpital Louis Mourier, Université René Descartes, Paris, France \\ chirdent@hotmail.com
}

VIH et troubles de l'hémostase sont particulièrement liés. En effet, l'infection par le VIH entraîne souvent une thrombopénie parfois importante et qui peut être révélatrice de l'infection (Godeau et Bierling 2004). Il s'agit d'un trouble périphérique lié à une hyperconsomation splénique des plaquettes : les mécanismes impliqués associent des dépôts spécifiques de complexes immuns et d'auto-anticorps.

Le VHC peut lui aussi provoquer des troubles de la coagulation : au stade d'insuffisance hépatique, il y a une insuffisance de production ou la synthèse de facteurs de coagulation anormaux, des anomalies quantitatives et qualitatives des plaquettes, une augmentation de l'activité fibrinolytique circulante, et la possibilité de survenue d'un processus de coagulation intravasculaire disséminée (CIVD) (Desconclois et Denninger 2010).

Ainsi, la co-infection VHC-VIH peut entraîner des risques hémorragiques majeurs.

Dans le Service d'Odontologie de l'Hôpital Louis Mourier (Colombes), nous avons été confrontés à plusieurs patients présentant une co-infection $\mathrm{VHC}-\mathrm{VIH}$, certains ayant un trouble majeur qui a nécessité une transfusion sanguine préopératoire.

Un protocole de prise en charge de ces patients permet, après évaluation du risque hémorragique, de déterminer les moyens d'hémostase à mettre en place et la nécessité ou non de transfusion de sang dans les cas les plus graves. (AFASSAPS 2003, Muller 2003, Arnaud et Simeoni 2006).

This is an Open Access article distributed under the terms of the Creative Commons Attribution-Noncommercial License 3.0, which permits unrestricted use, distribution, and reproduction in any noncommercial medium, provided the original work is properly cited. 